\title{
Lorentz violation and short-baseline neutrino experiments
}

\author{
V. Alan Kostelecký and Matthew Mewes \\ Physics Department, Indiana University, Bloomington, IN 47405, U.S.A.
}

(Dated: IUHET 470, June 2004)

\begin{abstract}
A general discussion is given of signals for broken Lorentz symmetry in short-baseline neutrino experiments. Among the effects that Lorentz violation can introduce are a dependence on energy differing from that of the usual massive-neutrino solution and a dependence on the direction of neutrino propagation. Using the results of the LSND experiment, explicit analysis of the effects of broken Lorentz symmetry yields a nonzero value $(3 \pm 1) \times 10^{-19} \mathrm{GeV}$ for a combination of coefficients for Lorentz violation. This lies in the range expected for effects originating from the Planck scale in an underlying unified theory.
\end{abstract}

Evidence for neutrino oscillations from the Liquid Scintillator Neutrino Detector (LSND) experiment remains one of the unresolved anomalies in neutrino physics $[1,2]$. The results of this experiment are consistent with muon antineutrinos $\bar{\nu}_{\mu}$ oscillating into electron antineutrinos $\bar{\nu}_{e}$ with a small probability of $P_{\bar{\nu}_{\mu} \rightarrow \bar{\nu}_{e}} \simeq 0.26 \pm 0.08 \%$ as the neutrinos propagate over a distance $L \simeq 30 \mathrm{~m}$. Typically, the oscillations are explained by ascribing small masses to neutrinos. The anomaly exists because the LSND result contradicts other neutrino-oscillation experiments in analyses based on three massive neutrinos [3]. More exotic oscillation scenarios may provide reconciliation of this contradiction. In the present work, we consider the possibility that the anomalous oscillations are induced by violation of Lorentz symmetry. We show that the size of the violation required to account for the anomaly is consistent with the size of effects that would emerge from an underlying unified theory at the Planck scale.

The principle of Lorentz symmetry is deeply ingrained in our best theories of nature at the fundamental level, which are Einstein's general relativity and the Standard Model of particle physics. The former accounts for gravitation at the classical level, while the latter describes all other physical phenomena involving elementary particles and forces down to the quantum level. However, a unification of these two theories is expected to occur at the Planck scale, $m_{P} \simeq 10^{19} \mathrm{GeV}$, about 17 orders of magnitude above the electroweak scale. Among the candidate experimental signals of new physics arising from this underlying unified theory are minuscule violations of Lorentz symmetry $[4,5]$.

Despite an expected suppression by $10^{-17}$ or more, Lorentz violation may be detectable with existing technology in experiments on a variety of systems, including neutrinos [6-15]. Under favorable circumstances, attainable sensitivities in the neutrino sector are competitive with those in other Lorentz tests, including ones with mesons [16], baryons [17], electrons [18-20], photons [2124], muons [25], and the Higgs [26]. Indeed, the current evidence for neutrino oscillations is compatible with small Lorentz violation, perhaps even without the introduction of neutrino mass [12]. The novel features introduced by Lorentz violation may also make possible a reconciliation of the LSND result with other neutrino experiments. The confirmation of oscillations from Lorentz violation in LSND and other neutrino experiments would offer the first glimpse of nature at its most basic level.

General Lorentz violation is described by a theoretical framework known as the Standard-Model Extension (SME) [6, 27]. It connects to the Planck scale through operators of nonrenormalizable dimension [28]. In the minimal form of the SME, neutrinos are massless and the oscillations are determined by a set of 102 real constant coefficients controlling the Lorentz violation [12]. These coefficients can be collected into matrices $\left(a_{L}\right)^{\mu}$ and $\left(c_{L}\right)^{\mu \nu}$ of mass dimension 1 and 0 , respectively, corresponding to CPT-odd and CPT-even operators. Setting all the coefficients to zero yields the usual minimal Standard Model of particle physics. Assuming quantumgravity origins for $\left(a_{L}\right)^{\mu}$ and $\left(c_{L}\right)^{\mu \nu}$ suggests their magnitude is suppressed by a factor of $10^{-17}$ or more.

The coefficients for Lorentz violation predict a plethora of unconventional signals in oscillation experiments. For example, the usual mass-induced oscillations vary as $L / E$ with the neutrino energy $E$ and the experimental baseline $L$. In contrast, the simplest Lorentz-violating oscillations vary as $L$ or $E L$, and more complicated functional dependence on $E$ can occur. This unconventional energy dependence can help reconcile conflicts with other experiments. For instance, within the massive-neutrino scenario the evidence for oscillations in LSND implies that the lower-energy CHOOZ experiment [29] should also have observed oscillations, contrary to the experimental results. However, the SME contains coefficients that predict oscillations in LSND without significant oscillations in CHOOZ [12].

Another unconventional feature of oscillations due to Lorentz violation is dependence on the direction of neutrino propagation. This causes several novel effects, which can include varying oscillation signals as the Earth rotates. For experiments like LSND, where both source and detector are fixed on the Earth's surface, the entire apparatus makes a full rotation each sidereal day $\simeq 23$ h $56 \mathrm{~min}$. The direction of propagation of the detected neutrinos is therefore also changing, and in the presence of Lorentz violation the consequent change in oscillation probabilities provides a definite signal that cannot arise from mass-induced oscillations [12]. This effect provides another path to reconciling the apparent conflict of LSND with other experiments. Thus, for example, LSND and 
the KARMEN experiment [30] conflict in the massiveneutrino case. However, the neutrinos in these two experiments propagate in different directions, so they can indeed behave differently in Lorentz-violating scenarios.

A more exotic possibility, absent in the minimal form of the SME but allowed in the general SME framework, is oscillations between neutrinos and antineutrinos [12]. Oscillations with $\nu \leftrightarrow \bar{\nu}$ mixing offer alternative Lorentzviolating modes that could explain the excess of $\bar{\nu}_{e}$ observed by LSND, since the numbers of $\nu_{e}, \nu_{\mu}$, and $\bar{\nu}_{\mu}$ involved are comparable. In what follows, we restrict attention to the minimal SME scenario.

The large number of coefficients involved, even in the minimal SME, makes a general analysis challenging. However, in experiments like LSND, the short baseline offers the possibility of a valuable simplifying approximation. When the baseline is small compared to the oscillation lengths given by the hamiltonian, the transition amplitudes can be expanded about the identity as a perturbation on the oscillation-free case. It turns out that the general leading-order result for the corresponding transition probabilities differs from the oscillationfree case by terms proportional to the squared modulus of hamiltonian elements, as we show next.

In the minimal Standard-Model Extension, the oscillatory behavior of the three generations of left-handed neutrinos is governed by the leading-order effective hamiltonian $[12]$

$$
h_{\mathrm{eff}}=\frac{1}{E}\left[\left(a_{L}\right)^{\mu} p_{\mu}-\left(c_{L}\right)^{\mu \nu} p_{\mu} p_{\nu}\right] .
$$

In this equation, $\left(a_{L}\right)^{\mu}$ and $\left(c_{L}\right)^{\mu \nu}$ are coefficients for Lorentz violation that are hermitian $3 \times 3$ complex matrices of mass dimension 1 and 0 , respectively. The energy $E$ is assumed to be large compared to the elements of $\left(a_{L}\right)^{\mu}$ and $E\left(c_{L}\right)^{\mu \nu}$. The four-momentum $p_{\mu} \simeq E(1 ;-\hat{p})$ introduces both energy dependence through $E$ and direction dependence through $\hat{p}$. Since the antisymmetric and trace pieces of $\left(c_{L}\right)^{\mu \nu}$ do not contribute to Eq. (1), we also assume in what follows the properties $\left(c_{L}\right)^{\mu \nu}=\left(c_{L}\right)^{\nu \mu}$ and $\eta_{\mu \nu}\left(c_{L}\right)^{\mu \nu}=0$. The effective hamiltonian for antineutrinos is obtained by complex conjugating Eq. (1) and reversing the sign of the $\left(a_{L}\right)^{\mu}$ term.

Under suitable experimental conditions, it is an excellent approximation to expand the oscillation amplitudes in powers of $h_{\text {eff }}: S(L) \simeq 1-i h_{\text {eff }} L /(\hbar c)-\frac{1}{2} h_{\text {eff }}^{2} L^{2} /(\hbar c)^{2}+$ $\cdots$. The validity of this expansion requires that the baseline $L$ be short compared to the oscillation lengths given by $h_{\text {eff }}$. However, since $h_{\text {eff }}$ varies with the neutrino energy $E$, the designation of a given experiment as short baseline in this context depends on the ranges of both $L$ and $E$. At leading order in this short-baseline approximation, the oscillation probabilities are

$$
P_{\nu_{b} \rightarrow \nu_{a}} \simeq \begin{cases}1-\sum_{c, c \neq a} P_{\nu_{a} \rightarrow \nu_{c}}, & a=b, \\ \left|\left(h_{\mathrm{eff}}\right)_{a b}\right|^{2} L^{2} /(\hbar c)^{2}, & a \neq b,\end{cases}
$$

where the indices $a, b$ range over the neutrino flavors $e, \mu, \tau$. The probabilities $P_{\bar{\nu}_{b} \rightarrow \bar{\nu}_{a}}$ for antineutrinos are obtained by changing the sign of $\left(a_{L}\right)^{\mu}$. Note that Eq. (2) can readily be modified for the nonminimal SME, including $\nu \leftrightarrow \bar{\nu}$ mixing [12].

In reporting results from experimental tests of Lorentz invariance, it is necessary to specify the frame of reference. In principle, any inertial frame can be adopted, but convention and convenience dictate the use of a Suncentered celestial-equatorial frame. For experiments with both source and detector fixed on the Earth's surface, the sidereal rotation causes the direction of neutrino propagation $\hat{p}$ to change with respect to the Sun-centered frame. This causes the components of $\hat{p}$ to vary at the sidereal frequency $\omega_{\oplus}=2 \pi /(23 \mathrm{~h} 56 \mathrm{~min})$, unless $\hat{p}$ happens to point along the Earth's rotation axis. This time dependence can be displayed explicitly in the effective hamiltonian $h_{\text {eff }}$, which can be written in the form

$$
\begin{aligned}
\left(h_{\mathrm{eff}}\right)_{a b}= & (\mathcal{C})_{a b}+\left(\mathcal{A}_{s}\right)_{a b} \sin \omega_{\oplus} T_{\oplus}+\left(\mathcal{A}_{c}\right)_{a b} \cos \omega_{\oplus} T_{\oplus} \\
& +\left(\mathcal{B}_{s}\right)_{a b} \sin 2 \omega_{\oplus} T_{\oplus}+\left(\mathcal{B}_{c}\right)_{a b} \cos 2 \omega_{\oplus} T_{\oplus},
\end{aligned}
$$

where $T_{\oplus}$ is the time measured from a standard origin [23]. This expression is independent of the short-baseline approximation, so Eq. (3) and what follows also apply more generally.

The energy dependence in Eq. (3) is given by further decomposition:

$$
\begin{aligned}
(\mathcal{C})_{a b} & =\left(\mathcal{C}^{(0)}\right)_{a b}+E\left(\mathcal{C}^{(1)}\right)_{a b} \\
\left(\mathcal{A}_{s}\right)_{a b} & =\left(\mathcal{A}_{s}^{(0)}\right)_{a b}+E\left(\mathcal{A}_{s}^{(1)}\right)_{a b} \\
\left(\mathcal{A}_{c}\right)_{a b} & =\left(\mathcal{A}_{c}^{(0)}\right)_{a b}+E\left(\mathcal{A}_{c}^{(1)}\right)_{a b} \\
\left(\mathcal{B}_{s}\right)_{a b} & =E\left(\mathcal{B}_{s}^{(1)}\right)_{a b}, \quad\left(\mathcal{B}_{c}\right)_{a b}=E\left(\mathcal{B}_{c}^{(1)}\right)_{a b} .
\end{aligned}
$$

The combinations $\left(\mathcal{A}_{s}^{(0)}\right)_{a b}, \quad\left(\mathcal{A}_{c}^{(0)}\right)_{a b}, \quad\left(\mathcal{C}^{(0)}\right)_{a b}$ contain the coefficients $\left(a_{L}\right)^{\mu}$, while $\left(\mathcal{A}_{s}^{(1)}\right)_{a b},\left(\mathcal{A}_{c}^{(1)}\right)_{a b},\left(\mathcal{B}_{s}^{(1)}\right)_{a b}$, $\left(\mathcal{B}_{c}^{(1)}\right)_{a b}, \quad\left(\mathcal{C}^{(1)}\right)_{a b}$ involve the coefficients $\left(c_{L}\right)^{\mu \nu}$. The analogous decomposition for the antineutrino effective hamiltonian generates combinations that can be obtained from their neutrino equivalents by complex conjugation and a sign reversal for $\left(\mathcal{A}_{s}^{(0)}\right)_{a b},\left(\mathcal{A}_{c}^{(0)}\right)_{a b},\left(\mathcal{C}^{(0)}\right)_{a b}$.

The explicit relationships between these quantities and the SME coefficients $\left(a_{L}\right)^{\mu}$ and $\left(c_{L}\right)^{\mu \nu}$ for Lorentz violation are

$$
\begin{aligned}
\left(\mathcal{C}^{(0)}\right)_{a b}= & \left(a_{L}\right)_{a b}^{T}-\hat{N}^{Z}\left(a_{L}\right)_{a b}^{Z}, \\
\left(\mathcal{C}^{(1)}\right)_{a b}= & -\frac{1}{2}\left(3-\hat{N}^{Z} \hat{N}^{Z}\right)\left(c_{L}\right)_{a b}^{T T}+2 \hat{N}^{Z}\left(c_{L}\right)_{a b}^{T Z} \\
& +\frac{1}{2}\left(1-3 \hat{N}^{Z} \hat{N}^{Z}\right)\left(c_{L}\right)_{a b}^{Z Z}, \\
\left(\mathcal{A}_{s}^{(0)}\right)_{a b}= & \hat{N}^{Y}\left(a_{L}\right)_{a b}^{X}-\hat{N}^{X}\left(a_{L}\right)_{a b}^{Y}, \\
\left(\mathcal{A}_{s}^{(1)}\right)_{a b}= & -2 \hat{N}^{Y}\left(c_{L}\right)_{a b}^{T X}+2 \hat{N}^{X}\left(c_{L}\right)_{a b}^{T Y} \\
& +2 \hat{N}^{Y} \hat{N}^{Z}\left(c_{L}\right)_{a b}^{X Z}-2 \hat{N}^{X} \hat{N}^{Z}\left(c_{L}\right)_{a b}^{Y Z}, \\
\left(\mathcal{A}_{c}^{(0)}\right)_{a b}= & -\hat{N}^{X}\left(a_{L}\right)_{a b}^{X}-\hat{N}^{Y}\left(a_{L}\right)_{a b}^{Y}, \\
\left(\mathcal{A}_{c}^{(1)}\right)_{a b}= & 2 \hat{N}^{X}\left(c_{L}\right)_{a b}^{T X}+2 \hat{N}^{Y}\left(c_{L}\right)_{a b}^{T Y} \\
& -2 \hat{N}^{X} \hat{N}^{Z}\left(c_{L}\right)_{a b}^{X Z}-2 \hat{N}^{Y} \hat{N}^{Z}\left(c_{L}\right)_{a b}^{Y Z},
\end{aligned}
$$




$$
\begin{aligned}
\left(\mathcal{B}_{s}^{(1)}\right)_{a b}= & \hat{N}^{X} \hat{N}^{Y}\left(\left(c_{L}\right)_{a b}^{X X}-\left(c_{L}\right)_{a b}^{Y Y}\right) \\
& -\left(\hat{N}^{X} \hat{N}^{X}-\hat{N}^{Y} \hat{N}^{Y}\right)\left(c_{L}\right)_{a b}^{X Y}, \\
\left(\mathcal{B}_{c}^{(1)}\right)_{a b}= & -\frac{1}{2}\left(\hat{N}^{X} \hat{N}^{X}-\hat{N}^{Y} \hat{N}^{Y}\right)\left(\left(c_{L}\right)_{a b}^{X X}-\left(c_{L}\right)_{a b}^{Y Y}\right) \\
& -2 \hat{N}^{X} \hat{N}^{Y}\left(c_{L}\right)_{a b}^{X Y} .
\end{aligned}
$$

In these expressions, $\hat{N}^{X}, \hat{N}^{Y}, \hat{N}^{Z}$ are directional factors containing information about the neutrino-beam direction with respect to the Earth. At the detector location, let $\theta$ be the angle between the beam and the vertical upward direction, let $\phi$ be the angle between the beam and south measured towards the east, and let $\chi$ be the colatitude of the detector. Then, the directional factors are given explicitly as

$$
\left(\begin{array}{c}
\hat{N}^{X} \\
\hat{N}^{Y} \\
\hat{N}^{Z}
\end{array}\right)=\left(\begin{array}{c}
\cos \chi \sin \theta \cos \phi+\sin \chi \cos \theta \\
\sin \theta \sin \phi \\
-\sin \chi \sin \theta \cos \phi+\cos \chi \cos \theta
\end{array}\right) .
$$

Any given short-baseline experiment is sensitive to three complex combinations of $\left(a_{L}\right)^{\mu}$ coefficients, $\left(\mathcal{A}_{s}^{(0)}\right)_{a b},\left(\mathcal{A}_{c}^{(0)}\right)_{a b},\left(\mathcal{C}^{(0)}\right)_{a b}$, and five complex combinations of $\left(c_{L}\right)^{\mu \nu}$ coefficients, $\left(\mathcal{A}_{s}^{(1)}\right)_{a b},\left(\mathcal{A}_{c}^{(1)}\right)_{a b},\left(\mathcal{B}_{s}^{(1)}\right)_{a b}$, $\left(\mathcal{B}_{c}^{(1)}\right)_{a b},\left(\mathcal{C}^{(1)}\right)_{a b}$. However, the directional dependence implies that a combination of experiments testing a specific oscillation mode $\nu_{a} \rightarrow \nu_{b}$ can provide access to all components of $\left(a_{L}\right)_{a b}^{\mu}$ and $\left(c_{L}\right)_{a b}^{\mu \nu}$, provided the directions of the associated neutrino beams differ.

For the special case of the transition mode relevant to LSND, the probability takes the form

$$
\begin{aligned}
P_{\bar{\nu}_{\mu} \rightarrow \bar{\nu}_{e}} \simeq \frac{L^{2}}{(\hbar c)^{2}} \mid(\mathcal{C})_{\bar{e} \bar{\mu}} \\
\quad+\left(\mathcal{A}_{s}\right)_{\bar{e} \bar{\mu}} \sin \omega_{\oplus} T_{\oplus}+\left(\mathcal{A}_{c}\right)_{\bar{e} \bar{\mu}} \cos \omega_{\oplus} T_{\oplus} \\
\quad+\left(\mathcal{B}_{s}\right)_{\bar{e} \bar{\mu}} \sin 2 \omega_{\oplus} T_{\oplus}+\left.\left(\mathcal{B}_{c}\right)_{\bar{e} \bar{\mu}} \cos 2 \omega_{\oplus} T_{\oplus}\right|^{2}
\end{aligned}
$$

where $\omega_{\oplus} \simeq 2 \pi /(23 \mathrm{~h} 56 \mathrm{~min})$ is the Earth's sidereal frequency and $T_{\oplus}$ is a standardized time [23]. The time variation is a direct consequence of the directional dependence. In the short-baseline approximation, we find harmonics up to $2 \omega_{\oplus}$, but more generally all higher harmonics can occur.

In Eq. (14), the complex factors $\left(\mathcal{A}_{s}\right)_{\bar{e} \bar{\mu}},\left(\mathcal{A}_{c}\right)_{\bar{e} \bar{\mu}}$, $\left(\mathcal{B}_{s}\right)_{\bar{e} \bar{\mu}},\left(\mathcal{B}_{c}\right)_{\bar{e} \bar{\mu}}$, and $(\mathcal{C})_{\bar{e} \bar{\mu}}$ are experiment-dependent linear combinations of the SME coefficients $\left(a_{L}\right)^{\mu}$ and $\left(c_{L}\right)^{\mu \nu}$ for Lorentz violation. These combinations depend on the energy of the neutrinos. Their decomposition into energy-independent quantities takes a form analogous to that of Eq. (4):

$$
\begin{aligned}
(\mathcal{C})_{\bar{e} \bar{\mu}} & =\left(\mathcal{C}^{(0)}\right)_{\bar{e} \bar{\mu}}+E\left(\mathcal{C}^{(1)}\right)_{\bar{e} \bar{\mu}} \\
\left(\mathcal{A}_{s}\right)_{\bar{e} \bar{\mu}} & =\left(\mathcal{A}_{s}^{(0)}\right)_{\bar{e} \bar{\mu}}+E\left(\mathcal{A}_{s}^{(1)}\right)_{\bar{e} \bar{\mu}} \\
\left(\mathcal{A}_{c}\right)_{\bar{e} \bar{\mu}} & =\left(\mathcal{A}_{c}^{(0)}\right)_{\bar{e} \bar{\mu}}+E\left(\mathcal{A}_{c}^{(1)}\right)_{\bar{e} \bar{\mu}} \\
\left(\mathcal{B}_{s}\right)_{\bar{e} \bar{\mu}} & =E\left(\mathcal{B}_{s}^{(1)}\right)_{\bar{e} \bar{\mu}}, \quad\left(\mathcal{B}_{c}\right)_{\bar{e} \bar{\mu}}=E\left(\mathcal{B}_{c}^{(1)}\right)_{\bar{e} \bar{\mu}} .
\end{aligned}
$$

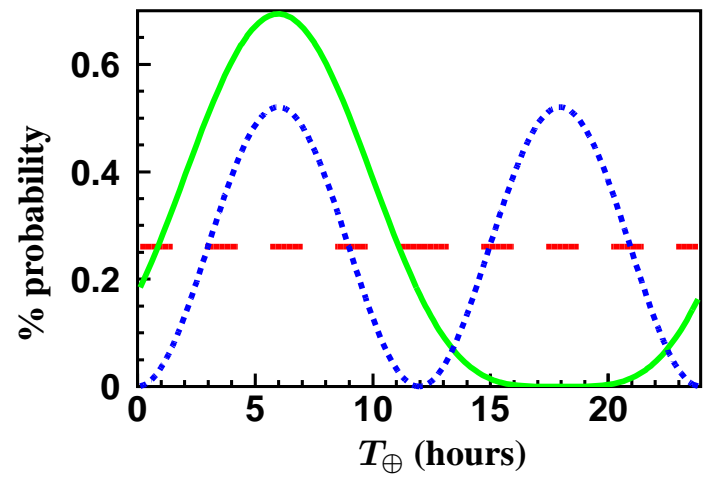

FIG. 1: Variations of the percent probability $P_{\bar{\nu}_{\mu} \rightarrow \bar{\nu}_{e}}$ over one sidereal day for three sample configurations with averaged probability $\left\langle P_{\bar{\nu}_{\mu} \rightarrow \bar{\nu}_{e}}\right\rangle=0.26 \%:(\mathcal{C})_{\bar{e} \bar{\mu}} \neq 0$ (dashed), $\left(\mathcal{A}_{s}\right)_{\bar{e} \bar{\mu}} \neq$ 0 (dotted), and $(\mathcal{C})_{\bar{e} \bar{\mu}}=\left(\mathcal{A}_{s}\right)_{\bar{e} \bar{\mu}} \neq 0$ (solid).

There are therefore a total of eight complex experimentdependent coefficients: $\left(\mathcal{A}_{s}^{(0)}\right)_{\bar{e} \bar{\mu}}, \quad\left(\mathcal{A}_{c}^{(0)}\right)_{\bar{e} \bar{\mu}}, \quad\left(\mathcal{C}^{(0)}\right)_{\bar{e} \bar{\mu}}$, $\left(\mathcal{A}_{s}^{(1)}\right)_{\bar{e} \bar{\mu}},\left(\mathcal{A}_{c}^{(1)}\right)_{\bar{e} \bar{\mu}},\left(\mathcal{B}_{s}^{(1)}\right)_{\bar{e} \bar{\mu}},\left(\mathcal{B}_{c}^{(1)}\right)_{\bar{e} \bar{\mu}},\left(\mathcal{C}^{(1)}\right)_{\bar{e} \bar{\mu}}$. A comprehensive analysis of the LSND data for the above energy and sidereal dependence would in principle yield measurements of 16 of the possible 102 real degrees of freedom in the neutrino sector of the minimal SME. We remark in passing that the inclusion of a mass-squared matrix $\left(\tilde{m}^{2}\right)_{a b}$ for neutrinos in the present formalism is straightforward. For example, in Eq. (15) it suffices to extend the definition of $(\mathcal{C})_{\bar{e} \bar{\mu}}$ to $(\mathcal{C})_{\bar{e} \bar{\mu}}=(2 E)^{-1}\left(\tilde{m}^{2}\right)_{\bar{e} \bar{\mu}}^{*}+$ $\left(\mathcal{C}^{(0)}\right)_{\bar{e} \bar{\mu}}+E\left(\mathcal{C}^{(1)}\right)_{\bar{e} \bar{\mu}}$. It turns out that the general twogeneration model with a mass-squared matrix and both $\left(a_{L}\right)^{\mu}$ and $\left(c_{L}\right)^{\mu \nu}$ coefficients has 41 degrees of freedom, while its rotation-invariant restriction has eight [12].

The published results from LSND permit the extraction of a measurement for one combination of these degrees of freedom. In the experiment, copious numbers of $\bar{\nu}_{\mu}$ were produced. An excess of $\bar{\nu}_{e}$ over background was observed, which was interpreted as $\bar{\nu}_{\mu}$ oscillating into $\bar{\nu}_{e}$. The corresponding oscillation probability is $P_{\bar{\nu}_{\mu} \rightarrow \bar{\nu}_{e}} \simeq 0.26 \pm 0.08 \%$. Since this published result involves all events irrespective of sidereal time, it represents an average over the run time of the experiment. To a good approximation, it can be taken as representing the expectation over a sidereal day, $\left\langle P_{\bar{\nu}_{\mu} \rightarrow \bar{\nu}_{e}}\right\rangle \simeq 0.26 \pm 0.08 \%$. Using Eq. (14) and this result, we obtain a nonzero measurement for a combination of SME coefficients for Lorentz violation:

$$
\begin{aligned}
\left|(\mathcal{C})_{\bar{e} \bar{\mu}}\right|^{2}+\frac{1}{2}\left|\left(\mathcal{A}_{s}\right)_{\bar{e} \bar{\mu}}\right|^{2}+\frac{1}{2}\left|\left(\mathcal{A}_{c}\right)_{\bar{e} \bar{\mu}}\right|^{2} & +\frac{1}{2}\left|\left(\mathcal{B}_{s}\right)_{\bar{e} \bar{\mu}}\right|^{2}+\frac{1}{2}\left|\left(\mathcal{B}_{c}\right)_{\bar{e} \bar{\mu}}\right|^{2} \\
\simeq & \frac{(\hbar c)^{2}\left\langle P_{\bar{\nu}_{\mu} \rightarrow \bar{\nu}_{e}}\right\rangle}{L^{2}} \\
\simeq & \left((3 \pm 1) \times 10^{-19} \mathrm{GeV}\right)^{2} .
\end{aligned}
$$

Since the LSND neutrino energy lies in the range $10 \mathrm{MeV}$ $\lesssim E \lesssim 50 \mathrm{MeV}$, this result corresponds to values of the SME coefficients for Lorentz violation of order $10^{-19} \mathrm{GeV}$ 
for $\left(a_{L}\right)^{\mu}$ and $10^{-17}$ for $\left(c_{L}\right)^{\mu \nu}$. Remarkably, these values are in the range predicted for quantum-gravity effects.

Establishing which specific combinations of the 16 possible degrees of freedom could be predominantly responsible for the LSND signal is more challenging and requires information about sidereal and energy dependences. Figure 1 illustrates some of the various possibilities. The probability $P_{\bar{\nu}_{\mu} \rightarrow \bar{\nu}_{e}}$ is displayed as a function of sidereal time for three situations with distinct combinations of nonzero coefficients $(\mathcal{C})_{\bar{e} \bar{\mu}}$ and $\left(\mathcal{A}_{s}\right)_{\bar{e} \bar{\mu}}$. The probabilities differ in detail, but all yield the result (16).

Other short-baseline experiments [29-34] could perform similar analyses to obtain further information about the space of coefficients for Lorentz violation. If the above solution is confirmed, it would constitute a convincing signal for Lorentz violation and could offer the first experimental glimpse of Planck-scale physics.

This work was supported in part by D.O.E. grant DEFG02-91ER40661 and N.A.S.A. grants NAG8-1770 and NAG3-2194.
[1] LSND Collaboration, C. Athanassopoulos et al., Phys. Rev. Lett. 81, 1774 (1998).

[2] LSND Collaboration, A. Aguilar et al., Phys. Rev. D 64, 112007 (2001).

[3] R.D. McKeown and P. Vogel, Phys. Rep. 394, 315 (2004).

[4] V.A. Kostelecký and S. Samuel, Phys. Rev. D 39, 683 (1989); V.A. Kostelecký and R. Potting, Nucl. Phys. B 359, 545 (1991).

[5] For reviews of approaches to Lorentz and CPT violation, see, for example, V.A. Kostelecký, ed., CPT and Lorentz Symmetry II, World Scientific, Singapore, 2002.

[6] D. Colladay and V.A. Kostelecký, Phys. Rev. D 55, 6760 (1997); Phys. Rev. D 58, 116002 (1998).

[7] S. Coleman and S.L. Glashow, Phys. Rev. D 59, 116008 (1999).

[8] V. Barger et al., Phys. Rev. Lett. 85, 5055 (2000).

[9] J.N. Bahcall, V. Barger, and D. Marfatia, Phys. Lett. B 534, 114 (2002).

[10] I. Mocioiu and M. Pospelov, Phys. Lett. B 537, 114 (2002).

[11] A. de Gouvêa, Phys. Rev. D 66, 076005 (2002).

[12] V.A. Kostelecký and M. Mewes, Phys. Rev. D, in press (hep-ph/0308300); Phys. Rev. D 69, 016005 (2004).

[13] G. Lambiase, Phys. Lett. B 560, 1 (2003).

[14] S. Choubey and S.F. King, Phys. Lett. B 586, 353 (2004).

[15] A. Datta et al., hep-ph/0312027.

[16] KTeV Collaboration, H. Nguyen, in Ref. [5]; OPAL Collaboration, R. Ackerstaff et al., Z. Phys. C 76, 401 (1997); DELPHI Collaboration, M. Feindt et al., preprint DELPHI 97-98 CONF 80 (1997); BELLE Collaboration, K. Abe et al., Phys. Rev. Lett. 86, 3228 (2001); BaBar Collaboration, B. Aubert et al., hep-ex/0303043; FOCUS Collaboration, J.M. Link et al., Phys. Lett. B 556, 7 (2003); V.A. Kostelecký, Phys. Rev. Lett. 80, 1818 (1998); Phys. Rev. D 61, 016002 (2000); Phys. Rev. D 64, 076001 (2001).

[17] L.R. Hunter et al., in V.A. Kostelecký, ed., CPT and Lorentz Symmetry, World Scientific, Singapore, 1999; D. Bear et al., Phys. Rev. Lett. 85, 5038 (2000); D.F. Phillips et al., Phys. Rev. D 63, 111101 (2001); M.A. Humphrey et al., Phys. Rev. A 68, 063807 (2003); Phys. Rev. A 62, 063405 (2000); F. Canè et al., physics/0309070; V.A. Kostelecký and C.D. Lane, Phys. Rev. D 60, 116010 (1999); J. Math. Phys. 40, 6245 (1999); R. Bluhm et al., Phys. Rev. Lett. 88, 090801 (2002); Phys. Rev. D 68, 125008 (2003).

[18] H. Dehmelt et al., Phys. Rev. Lett. 83, 4694 (1999); R. Mittleman et al., Phys. Rev. Lett. 83, 2116 (1999); G. Gabrielse et al., Phys. Rev. Lett. 82, 3198 (1999); R.
Bluhm et al., Phys. Rev. Lett. 82, 2254 (1999); Phys. Rev. Lett. 79, 1432 (1997); Phys. Rev. D 57, 3932 (1998); D. Colladay and V.A. Kostelecký, Phys. Lett. B 511, 209 (2001); B. Altschul, hep-ph/0405084.

[19] B. Heckel, in Ref. [5]; L.-S. Hou, W.-T. Ni, and Y.-C.M. Li, Phys. Rev. Lett. 90, 201101 (2003); R. Bluhm and V.A. Kostelecký, Phys. Rev. Lett. 84, 1381 (2000).

[20] H. Müller et al., Phys. Rev. D 68, 116006 (2003).

[21] S.M. Carroll, G.B. Field, and R. Jackiw, Phys. Rev. D 41, 1231 (1990); M.P. Haugan and T.F. Kauffmann, Phys. Rev. D 52, 3168 (1995); V.A. Kostelecký and M. Mewes, Phys. Rev. Lett. 87, 251304 (2001).

[22] R. Jackiw and V.A. Kostelecký, Phys. Rev. Lett. 82, 3572 (1999); V.A. Kostelecký et al., Phys. Rev. D 65, 056006 (2002); C. Adam and F.R. Klinkhamer, Nucl. Phys. B 657, 214 (2003); V.A. Kostelecký, R. Lehnert, and M. Perry, Phys. Rev. D 68, 123511 (2003); H. Müller et al., Phys. Rev. D 67, 056006 (2003); T. Jacobson et al., Phys. Rev. D 67, 124011 (2003); V.A. Kostelecký and A.G.M. Pickering, Phys. Rev. Lett. 91, 031801 (2003); R. Lehnert, Phys. Rev. D 68, 085003 (2003); G.M. Shore, Contemp. Phys. 44, 503 2003; B. Altschul, hep-th/0311200; hep-th/0402036; R. Lehnert and R. Potting, hep-ph/0406128.

[23] V.A. Kostelecký and M. Mewes, Phys. Rev. D 66, 056005 (2002).

[24] J. Lipa et al., Phys. Rev. Lett. 90, 060403 (2003); H. Müller et al., Phys. Rev. Lett. 91, 020401 (2003); P. Wolf et al., gr-qc/0401017.

[25] V.W. Hughes et al., Phys. Rev. Lett. 87, 111804 (2001); R. Bluhm et al., Phys. Rev. Lett. 84, 1098 (2000).

[26] D.L. Anderson, M. Sher, and I. Turan, hep-ph/0403116; E.O. Iltan, hep-ph/0405119.

[27] V.A. Kostelecký, Phys. Rev. D 69, 105009 (2004).

[28] V.A. Kostelecký and R. Potting, Phys. Rev. D 51, 3923 (1995); V.A. Kostelecký and R. Lehnert, Phys. Rev. D 63, 065008 (2001).

[29] M. Apollonio et al., Phys. Lett. B 466, 415 (1999).

[30] KARMEN Collaboration, B. Armbruster et al., Phys. Rev. D 65, 112001 (2002).

[31] CHORUS Collaboration, E. Eskut et al., Phys. Lett. B 497, 8 (2001).

[32] MiniBooNE Collaboration, E. Church et al., Fermilab Report No. FERMILAB-P-0898, 1997.

[33] NOMAD Collaboration, P. Astier et al., Phys. Lett. B 570, 19 (2003).

[34] S. Avvakumov et al., Phys. Rev. Lett. 89, 011804 (2002). 